\title{
Estimación de abundancia y biomasa del cangrejo dorado (Chaceon chilensis), en el archipiélago de Juan Fernández, Chile*
}

\author{
Patricio M. Arana \\ Escuela de Ciencias del Mar, Universidad Católica de Valparaíso \\ Casilla 1020, Valparaíso, Chile \\ E-mail: parana@ucv.cl
}

Recibido: 15 enero 1999; versión corregida: 23 junio 1999; aceptado: 21 septiembre 1999

RESUMEN Se determinan aspectos biológico-pesqueros del cangrejo dorado de Juan Fernández (Chaceon chilensis), encontrado recientemente en torno a las islas Robinson Crusoe y Santa Clara (archipiélago de Juan Fernández, Chile), y se entrega una primera estimación de la abundancia y biomasa de este recurso. La información utilizada fue obtenida en muestreos efectuados en el marco de la pesca experimental de esta especie realizado entre 175 y $625 \mathrm{~m}$ de profundidad, de marzo a junio de 1997. Para la determinación de la abundancia y de la biomasa de este cangrejo se utilizó el área teórica de influencia de las trampas. Debido a incertidumbre en la estimación de los parámetros utilizados, alguno de ellos fueron sensibilizados dentro de ciertos márgenes con el objeto de obtener un rango de valores posibles. La captura C. chilensis se caracteriza por la presencia casi absoluta de machos $(97,8 \%)$, aspecto que es independiente del tipo de trampa utilizado y del rango batimétrico. Con relación a las tallas, los ejemplares estuvieron comprendidos entre 84 y $147 \mathrm{~mm}$ de longitud cefalotorácica. Respecto al peso total individual, los valores máximos y mínimos observados correspondieron respectivamente a 250 y $1410 \mathrm{~g}$, con un peso total promedio (machos) de $863 \mathrm{~g}$. Al efectuar una primera estimación de la biomasa de $C$. chilensis, sensibilizando para ello el área de influencia de la trampa, se determinó como biomasa disponible más probable 995,3 ton, lo que significa una densidad promedio de 2,9 individuos $/ 1000 \mathrm{~m}^{2}$ y un rendimiento máximo sostenible (RMS) más probable de 75 ton. En el escenario más pesimista, se obtiene un RMS de 19 ton, mientras que en el más optimista alcanza a 168 ton.

Palabras clave: cangrejo dorado, Chaceon chilensis, abundancia, biomasa, RMS, archipiélago de Juan Fernández, Chile.

\section{Abundance and biomass estimates of golden crab (Chaceon chilensis), in the Juan Fernandez archipelago, Chile}

\begin{abstract}
ABSIRACT. The fishery-biology aspects of the Juan Fernandez golden crab (Chaceon chilensis), and first estimates of the abundance and biomass of this resource distributed in the marine substrate surrounding Robinson Crusoe and Santa Clara islands are given. Information was obtained from samples taken within an experimental fishing survey for this crustacean, between 175 to $600 \mathrm{~m}$ depth, from March to June 1997. The theoretical area of influence methodology was used to estimate the abundance and biomass. Due to the degree of uncertainty of the variables employed, some parameters were sensibilized in order to obtain a range of estimates, along with its most likely value. Results indicate that the fishery of this resource is characterized by its catch mainly composed by males $(97,8 \%)$, in the different types of traps used as well as in the whole bathymetric range surveyed. Specimens' length were between 84 and $147 \mathrm{~mm}$ of cephalothoraxic length. Maximum and minimum observed total weights were 250 and $1410 \mathrm{~g}$ respectively. The mean weight of a specimen (male) was $863 \mathrm{~g}$. First most likely estimate of the golden crab available biomass, having sensibilized the area of influence of the trap, was determined to be 995,3 tons, that is a mean density of 2,9 individual/ $1000 \mathrm{~m}^{2}$ and a maximum sustainable yield (MSY) of 75 tons. In the worst of the estimates scenery a MSY of 19 tons was obtained, and in the most optimistic scenery it reached 168 tons.
\end{abstract}

Key words: golden crab, Chaceon chilensis, abundance, biomass, MSY, Juan Fernandez archipelago, Chile.

\footnotetext{
* Artículo generado como parte del "Programa de pesca exploratoria y experimental de recursos pesqueros alternativos a la langosta en las islas Robinson Crusoe y Santa Clara” (Proyecto FIP 95/25), financiado por el Fondo de Investigación Pesquera y realizado por la Universidad Católica de Valparaíso.
} 


\section{INTRODUCCIÓN}

El cangrejo dorado de Juan Fernández (Chaceon chilensis Chirino-Gálvez y Manning, 1989) es un crustáceo braquiuro descrito en aguas del Pacífico Suroriental. La clasificación taxonómica de este cangrejo fue redefinida por Chirino-Gálvez y Manning (1989), siendo previamente clasificado como Geryon quinquedens (Retamal, 1977, 1981; Andrade, 1985), y como G. affinis (Dupré, 1975; Báez y Andrade, 1977; Chirino-Gálvez, 1985 y Báez y Ruiz, 1985).

C. chilensis pertenece a la familia Geryonidae que según Dawson y Webber (1991) está compuesta por los géneros Geryon, Chaceon y Zariquieyon, que están representados por 4, 23 y 1 especies vivientes, respectivamente. En general, los ejemplares del género Geryon se conocen con el nombre común de "cangrejos rojos" o "red crabs" y los del género Chaceon como "cangrejos dorados" o "golden crabs". Algunos son explotados en diversos lugares del mundo, constituyendo pesquerías de cierta importancia, todas ellas destinadas a consumo humano. Son organismos de gran tamaño y peso, que habitan en aguas profundas.

Durante 1997, la Escuela de Ciencias del Mar de la Universidad Católica de Valparaíso desarrolló un programa de pesca exploratoria en el archipiélago de Juan Fernández, labor que fue financiada por el Fondo de Investigación Pesquera (FIP). El principal objetivo de dichas labores consistió en identificar recursos pesqueros potenciales, a fin de diversificar la pesquería existente en estas islas, la cual está orientada casi exclusivamente a la extracción de la langosta (Jasus frontalis H. Milne-Edwards, 1837). Como resultado se detectó la presencia del cangrejo dorado ( $C$. chilensis) en torno a ambas islas, entre 175 y $1000 \mathrm{~m}$ de profundidad (Arana, 2000). Esto motivó la realización de una campaña de pesca experimental, que fue orientada a determinar el aparejo más adecuado para su captura, definir la distribución en profundidad y establecer el equipamiento y régimen operacional apropiado para la extracción de este recurso (Arana y Vega, 2000).

El objetivo del presente es el establecer un primer estimado de la abundancia y biomasa de $C$. chilensis, en aguas circundantes a las islas Robinson Crusoe y Santa Clara. Así también, dada la importancia que puede representar esta especie para los pescadores insulares, se consideró la determinación de algunos aspectos básicos de su biología pesquera.

\section{MATERIALES Y MÉTODOS}

\section{Aspectos generales}

La información utilizada fue obtenida en las operaciones de pesca experimental del cangrejo dorado, realizadas entre 175 y $625 \mathrm{~m}$ de profundidad en aguas del archipiélago de Juan Fernández. La zona de estudio se ubicó al área noreste de la isla Robinson Crusoe, frente a bahía Cumberland (Fig. 1), en atención a que se determinó la presencia de esta especie en todos los fondos que fueron previamente explorados (Arana, 2000). El área proporciona la conveniencia adicional de estar cerca de la base de operaciones (San Juan Bautista), para permitir el rápido retorno a puerto ante eventuales cambios meteorológicos.

Las trampas empleadas incluyeron diversas variantes tecnológicas de diseño, dimensiones geométricas, así como forma y tamaño de las entradas. Los diseños utilizados fueron: a) Troncocónica con entrada rectangular; b) Troncocónica con entrada circular; c) Tetragonal pequeña con entrada rectangular; d) Tetragonal pequeña con entrada circular; e) Tetragonal grande con entrada rectangular; f) Tetragonal grande con entrada circular; g) Piramidal pequeña con entrada rectangular; h) Piramidal pequeña con entrada circular; i) Piramidal grande con entrada rectangular; j) Piramidal grande con entrada circular; k) Langostera con entrada en un extremo; y l) Langostera con entrada en la parte superior. Se trabajó simultáneamente con varias tenas compuestas cada una por dos trampas de distinto diseño, amarradas a la línea de calado a $100 \mathrm{~m}$ la una de la otra, con chicotes de $30 \mathrm{~m}$ de longitud. Las trampas fueron caladas mediante un orinque de polietileno torcido de 5 y $6 \mathrm{~mm}$ de diámetro. Como carnada se utilizaron especies de peces locales (Arana y Vega, 2000).

En la campaña de pesca experimental se utilizó la chalupa de alta mar "Miriam", que corresponde al diseño y dimensiones de las embarcaciones usadas por los pescadores artesanales en estas islas. Se llevó registro de fecha, posición geográfica, profundidad de trabajo, tipo de carnada utilizada, diseño de las trampas y captura en número en cada unidad experimental (trampa). Además, se determinó in situ el sexo y la longitud del caparazón (Lc) con un medidor vernier con precisión de $\pm 1 \mathrm{~mm}$ y el peso total de los ejemplares capturados con un dinamómetro. 


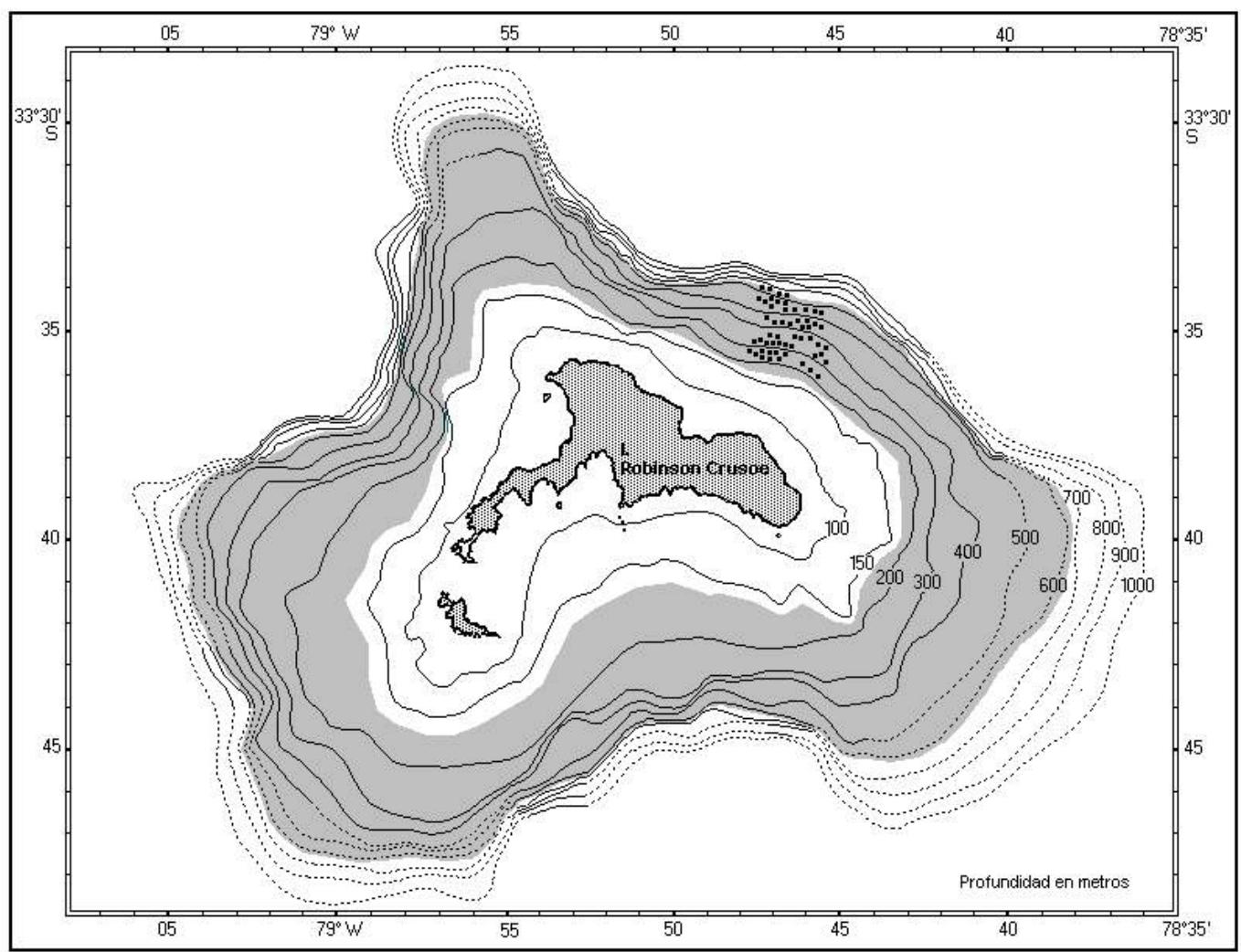

Figura 1. Ubicación de las trampas durante la pesca experimental (•) y área de estimación de la biomasa y abundancia (sombreado) del cangrejo dorado (Chaceon chilensis).

Figure 1. Location of traps $(\bullet)$ during the experimental fishing of the golden crab (Chaceon chilensis). Biomass and abundance area of estimation (gray area).

En tierra a una cierta cantidad de cangrejos, se les midió el largo (Lc) y ancho del caparazón (Ac) y el peso en una balanza digital con precisión de $\pm 1 \mathrm{~g}$. Con estos registros se determinó la relación entre el largo y el ancho cefalotóracico, así como también las relaciones entre longitud cefalotorácica (Lc) y peso total $(\mathrm{Wt})$ y entre ancho cefalotorácico (Ac) y peso total.

\section{Metodología de evaluación}

La estimación de abundancia se efectuó mediante la metodología del Area Teórica de Influencia de las Trampas. Este procedimiento, que se asemeja al de Areabarrida cuando se trabaja con redes de arrastre, se ha propuesto para evaluar poblaciones marinas en el caso de recursos que son capturados con trampas (Miller, 1975; McElman y Elner, 1982; Eggers et al., 1982; Boschi et al., 1984; MelvilleSmith, 1986; Arena et al., 1988). Este método ha sido empleado por la Comisión para la Conservación de los Recursos Vivos Marinos Antárticos en la primera estimación del tamaño del stock de Paralomis spinosissima Birstein y Vinogradov, 1972, en torno a la isla Georgia del Sur, como así también una adaptación de este método, para calcular la biomasa de Dissostichus eleginoides Smitt, 1998 en los alrededores de esa misma isla, utilizando para ello información de capturas realizadas con espineles de profundidad (CCRVMA, 1992).

La metodología de área de influencia de las trampas se sustenta en que los organismos son muy sensibles a los estímulos químicos de las sustancias disueltas en el agua por los cebos o carnadas. De allí que cada trampa tiene un "radio de acción", o "un área teórica de influencia" respecto a los organismos que se encuentran a su alrededor (Boschi et al., 1984; Arena et al., 1988). 


\section{Método de Area Teórica de Influencia de las Trampas}

Para obtener una estimación aproximada de la abundancia, se utilizó la siguiente expresión:

$$
\mathrm{Ab}=\mathrm{A}_{\mathrm{t}} * \mathrm{D}_{\mathrm{m}} * \mathrm{q}
$$

donde:

$\mathrm{Ab}$ : abundancia ( $\mathrm{N}^{\mathrm{o}}$ de individuos)

$A_{t}$ : área total de distribución del recurso $\left(\mathrm{mn}^{2}\right)$, en el rango batimétrico de su distribución

$\mathrm{D}_{\mathrm{m}}$ : densidad media del recurso ( $\mathrm{N}^{\mathrm{o}}$ de individuos/ $\mathrm{mn}^{2}$ )

q : Coeficiente de capturabilidad. Tasa de captura o proporción de individuos que se encuentran en el área de influencia de la trampa y que son efectivamente capturados por el aparejo.

A su vez, la densidad media se estimó de la siguiente manera:

donde:

$$
\mathrm{D}_{\mathrm{m}}=\frac{\mathrm{C}_{\mathrm{t}}}{\alpha}
$$

$\mathrm{C}_{\mathrm{t}}$ : captura promedio por trampa $\left(\mathrm{N}^{\mathrm{o}}\right.$ de individuos)

a : área teórica de influencia de la trampa $\left(\mathrm{mn}^{2}\right)$

Si se considera que el área teórica de influencia del aparejo presenta una forma circular, bajo el supuesto de mínima o nula corriente submarina (Eggers et al., 1982; Arena et al., 1988), entonces:

$$
\alpha=\pi * \mathrm{r}^{2}
$$

donde:

$\mathrm{r}$ : radio teórico de acción del aparejo $(\mathrm{mn})$

$\mathrm{Al}$ reemplazar estas expresiones en la primera ecuación, se tiene que la abundancia está representada por:

$$
\mathrm{Ab}=\mathrm{A}_{\mathrm{t}} * \frac{\mathrm{Ct}}{\pi * \mathrm{r}^{2}} * \mathrm{q}
$$

Si estos cálculos se realizan por estrato de profundidad o subárea, entonces la expresión anterior se transforma en:

$$
\mathrm{Ab}=\sum_{\mathrm{i}=1}^{\mathrm{n}} \mathrm{a}_{\mathrm{i}} *\left(\frac{\mathrm{C}_{\mathrm{i}}}{\pi * \mathrm{r}^{2}}\right) * \mathrm{q}
$$

donde:

$\mathrm{a}_{\mathrm{i}}$ : área del estrato o subárea (i)

$\mathrm{C}_{\mathrm{i}}$ : captura promedio por trampa en el estrato o subárea (i) ( $\mathrm{N}^{\mathrm{o}}$ de individuos)

i : estrato o subárea $(\mathrm{i}=1,2, \ldots, \mathrm{n})$

Para contar con un primer estimado del rendimiento máximo sostenible (RMS), se utilizó la fórmula propuesta para un stock virgen por Gulland (1971). Esta aproximación es útil en este caso, dado que es la primera vez que se intenta la captura del cangrejo dorado en este archipiélago. El RMS queda definido por la siguiente expresión:

$$
R M S=0,5 * M * B v
$$

donde:

$\mathrm{Bv}$ : biomasa del stock virgen (peso)

M : coeficiente de mortalidad natural (año ${ }^{-1}$ )

Debido a la incerteza de las variables utilizadas para el cálculo de abundancia y biomasa, se efectuó la sensibilización del área de influencia de la trampa (a) y del coeficiente de capturabilidad (q) dentro de un cierto rango, a fin de disponer de una gama de estimados, junto al valor considerado como más probable.

Con el fin de determinar el área de distribución del cangrejo dorado en torno a las islas, se midió la superficie del área de fondo marino sobre la carta batimétrica, realizándose esta tarea por estrato de profundidad cada $50 \mathrm{~m}$, entre $175 \mathrm{~m}$ y $625 \mathrm{~m}$ de profundidad, que fue el rango utilizado en la pesca experimental (Fig. 1), utilizando un planímetro polar.

\section{RESULTADOS}

\section{Aspectos generales}

Las faenas de pesca experimental se realizaron entre el 23 de marzo y el 2 de junio de 1997, en el rango entre 175 y $625 \mathrm{~m}$ de profundidad. La elección de dicho rango tiene relación con la distribución batimétrica del recurso objetivo (Arana, 2000; 
Arana y Vega, 2000) y la dificultad de operar a más de $600 \mathrm{~m}$ de profundidad con una embarcación artesanal.

\section{Composición de la población}

La capturas de $C$. chilensis se caracterizó por la extracción casi exclusiva de machos. En todo el período de trabajo, con todos los tipos de trampas utilizados y en todo el rango de profundidad estudiado la fracción de hembras alcanzó únicamente al 2,2\% ( $\mathrm{n}=56$ hembras). Esto motivó que los análisis biológico-pesqueros se hayan efectuado solamente en machos de esta especie.

Respecto al peso total individual, los valores máximos y mínimos observados correspondieron respectivamente a 250 y $1410 \mathrm{~g}$. El peso total promedio de los ejemplares (machos) fue de $863 \mathrm{~g}$.

Los ejemplares estuvieron comprendidos entre 84 y $147 \mathrm{~mm}$ de longitud cefalotorácica. La distribución de frecuencias de tallas de los machos es claramente polimodal, con la moda centrada alrededor de $118 \mathrm{~mm}$ de longitud cefalotorácica. Se destaca en ella la escasa proporción de ejemplares con ta- llas menores de $105 \mathrm{~mm}$ y mayores de $130 \mathrm{~mm}$ En el caso de las escasas hembras capturadas, se distribuyeron principalmente a los $85-100 \mathrm{~mm}, 105-110$ mm y $130-140$ mm. (Fig. 2).

Al comparar las tallas medias de C. chilensis capturados por rango de profundidad, éstas mostraron ser estadísticamente semejantes entre 400 y $550 \mathrm{~m}$ (Fig. 3). No obstante, los promedios determinados en 250, 300 y $350 \mathrm{~m}$, así como en $600 \mathrm{~m}$ son estadísticamente diferentes entre sí y mayores a los observados en el rango 400 a $550 \mathrm{~m}$. Esto demuestra que los ejemplares de mayor talla se encuentran distribuidos principalmente sobre $425 \mathrm{~m}$ y bajo $575 \mathrm{~m}$ de profundidad.

\section{Relaciones morfométricas}

Para convertir las medidas de largo del caparazón (Lc) en su correspondiente ancho de caparazón (Ac) y vice versa, se estableció la relación entre ambas variables mediante un ajuste regresional de tipo lineal (Fig. 4 y Tabla 1). Con este fin, se midieron 241 machos inmediatamente después de ser izados a bordo.
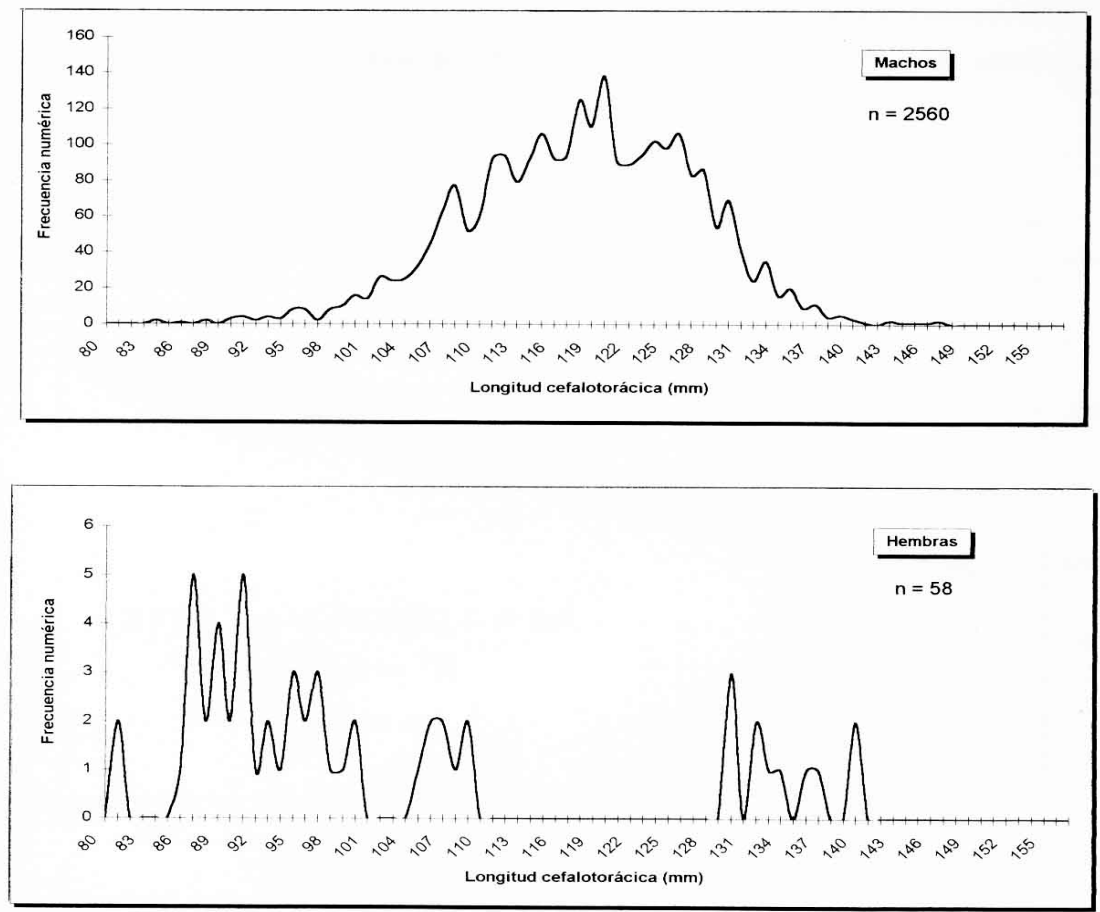

Figura 2. Distribución de frecuencias de tallas del cangrejo dorado (Chaceon chilensis). Figure 2. Length frequency distributions of golden crab (Chaceon chilensis). 


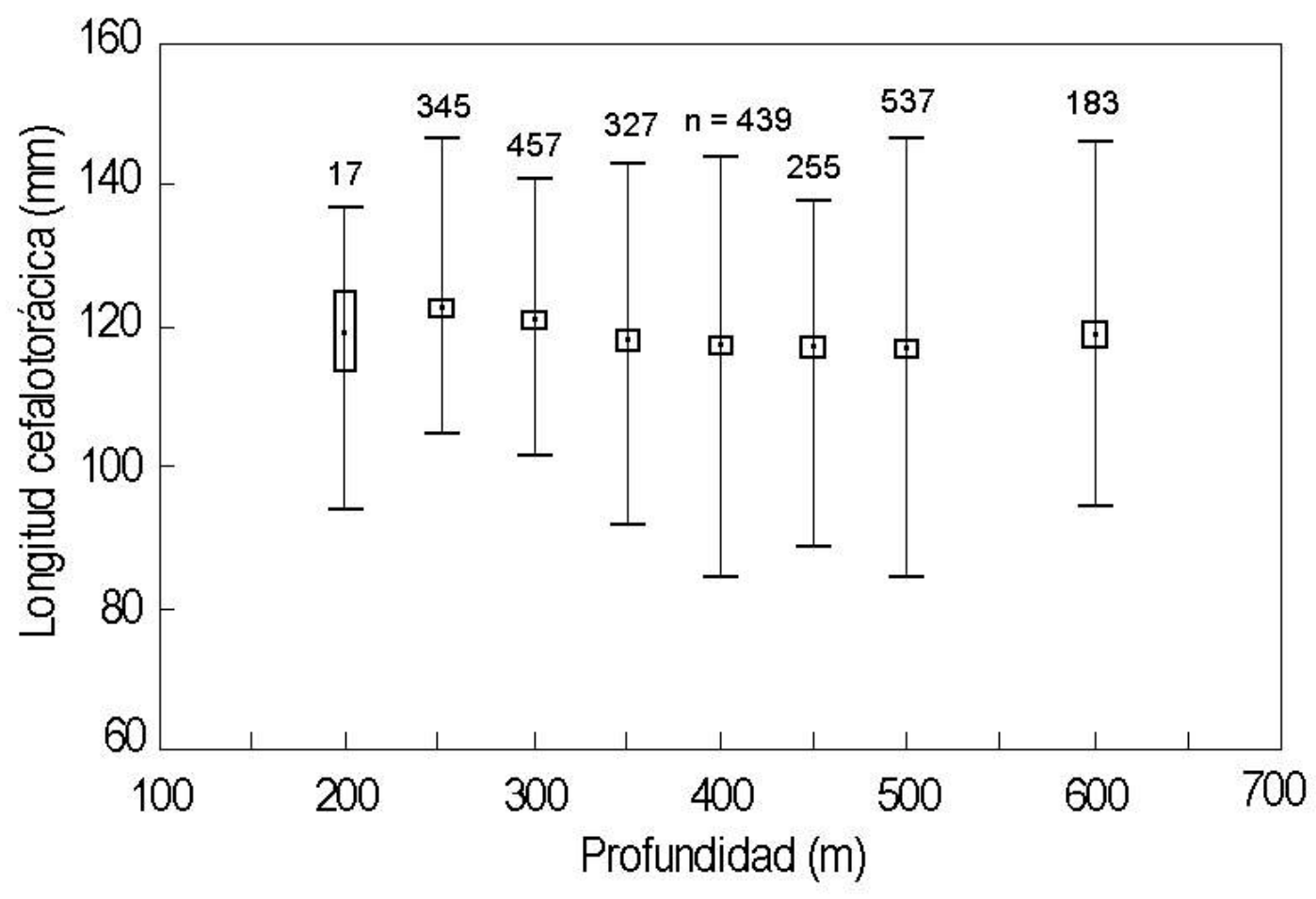

Figura 3. Longitudes cefalotorácicas medias, banda de confianza al $95 \%$ y desviación estándar de cangrejo dorado capturado a diferentes profundidades.

Figure 3. Cephalothoraxic mean lengths, $95 \%$ confidence band and standard deviation for golden crab caught at different depths.

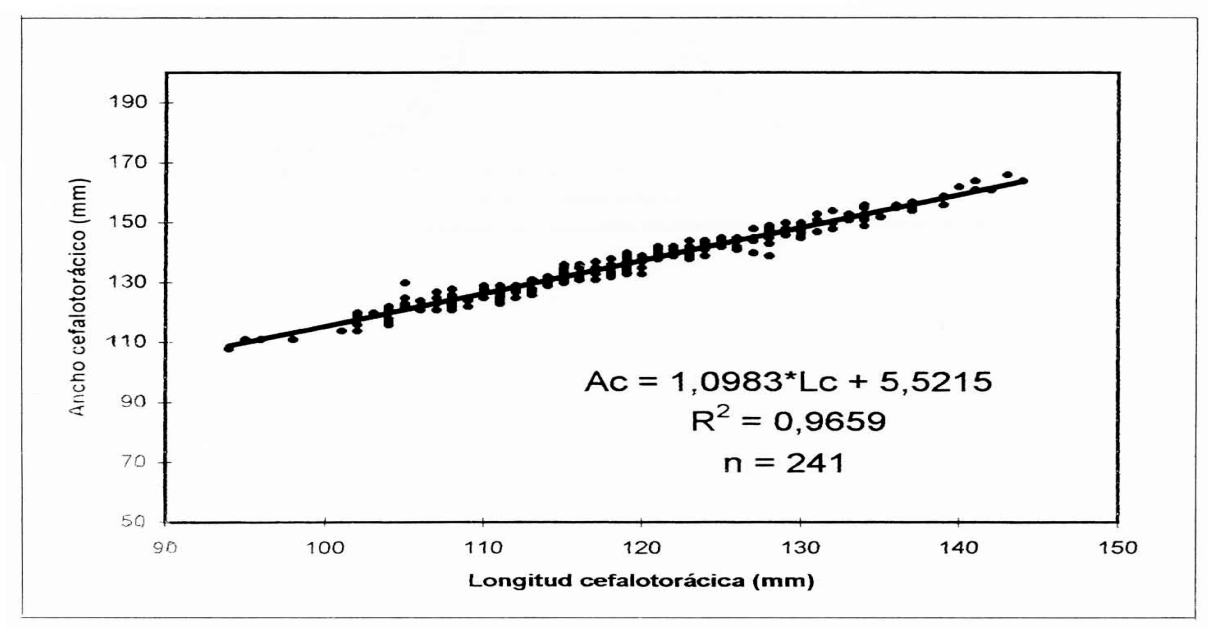

Figura 4. Relación entre la longitud y el ancho cefalotorácico en el cangrejo dorado (Chaceon chilensis). Figure 4. Relationship between length and cephalothoraxic width of golden crab (Chaceon chilensis). 
Igualmente, se estableció en los machos la relación entre la longitud cefalotorácica $(\mathrm{Lc})$ y el peso total húmedo (Wt), como así también el ancho cefalotorácico (Ac) vs el peso total húmedo. Con este fin se midieron y pesaron en tierra 594 ejemplares. En ambos casos, al correlacionar los valores mediante un ajuste de poder se encontró un valor de «b» aproximadamente igual a 3, razón por la cual se puede considerar dicha relación como isométrica (Tabla 1). Al mismo tiempo, en la relación Lc-Wt se observa una notoria heterocedasticidad en los valores, con un alto grado de variación en el peso determinado en los ejemplares de gran talla, que incluso puede llegar a diferencias de hasta un $20 \%$ respecto al peso medio calculado mediante la ecuación establecida con dicha relación (Fig. 5).

\section{Estimación de abundancia y biomasa}

Para determinar la biomasa disponible de $C$. chilensis, se consideraron los siguientes aspectos, a la vez que se determinaron los valores que se indi- can a continuación:

a) Distribución del recurso. Se consideró que la distribución del cangrejo dorado es similar al observado en el área donde se realizó la pesca experimental, dada las características de estas islas y los resultados obtenidos en las faenas de pesca exploratoria (Arana, 2000; Arana y Vega, 2000). Así también, si bien este crustáceo se encontró entre 175 y $1000 \mathrm{~m}$ de profundidad, la evaluación se efectuó únicamente hasta los 625 $\mathrm{m}$, dado que sólo hasta esa profundidad se contó con registros representativos de captura por unidad de esfuerzo.

b) Area de cada estrato de profundidad. El área total entre 175 y $625 \mathrm{~m}$ es de $133,2 \mathrm{mn}^{2}$ (Tabla 2). En el caso del estrato 176-225 m, se utilizó sólo la mitad del área determinada, en atención a que ésta corresponde al área límite de distribución del recurso. De esta forma, se considera que hasta $625 \mathrm{~m}$ el área total en que se encuentra presente este recurso es equivalente a $117,5 \mathrm{mn}^{2}$. c) Captura por trampa. Para la evaluación de la

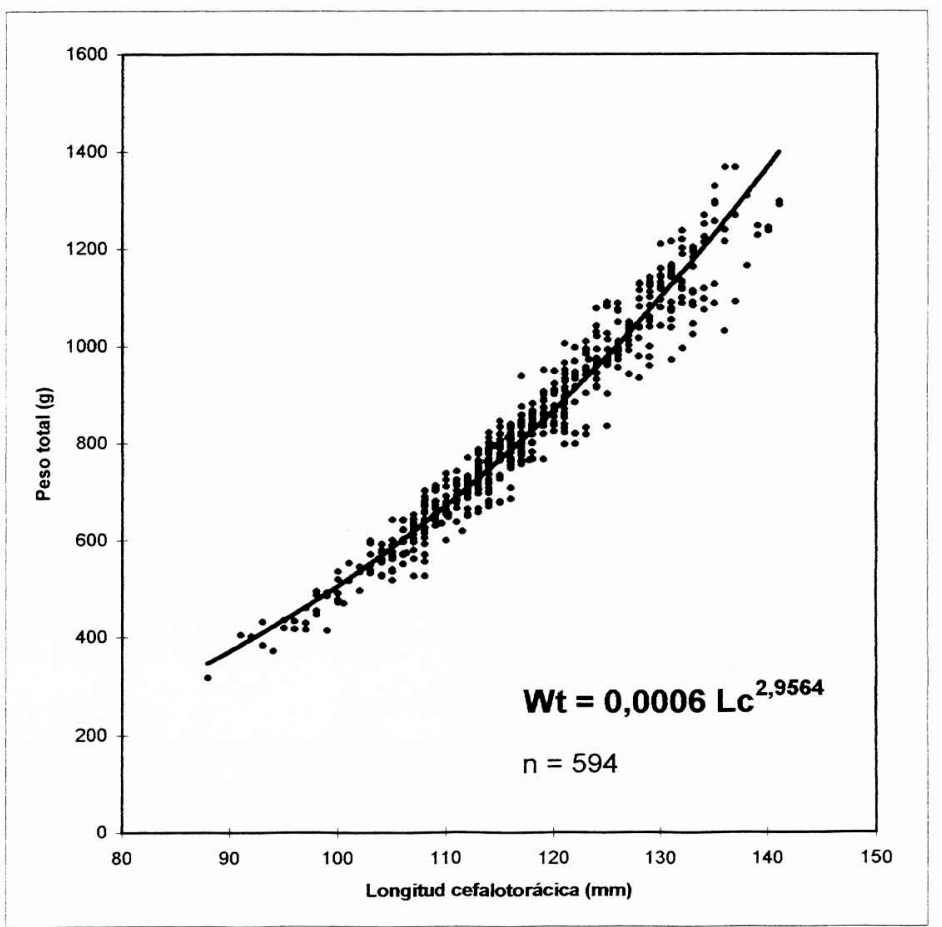

Figura 5. Relación talla-peso en el cangrejo dorado (Chaceon chilensis).

Figure 5. Length-weight relationship of golden crab (Chaceon chilensis). 
Tabla 1. Relaciones morfométricas establecidas en machos de cangrejo dorado (Chaceon chilensis) (*). Table 1. Male golden crab (Chaceon chilensis) morphometric relationships.

\begin{tabular}{|c|c|c|c|c|c|c|}
\hline \multirow[t]{2}{*}{ Relación } & \multirow[t]{2}{*}{ Ecuación } & \multirow{2}{*}{$\begin{array}{l}\text { Númer o } \\
\text { de } \\
\text { obser va- } \\
\text { ciones }\end{array}$} & \multirow{2}{*}{$\begin{array}{l}\text { Rango } \\
\text { de longitud } \\
\text { cefalotorá- } \\
\text { cica (mm) }\end{array}$} & \multicolumn{3}{|c|}{ Parámetr os } \\
\hline & & & & $\mathbf{a}$ & b & $\mathbf{r}^{2}$ \\
\hline Longitud (Lc) - ancho cefalotorácico (Ac) & $A c=a+b * L c$ & 241 & $94-144$ & 5,521 & 1,098 & 0,966 \\
\hline Ancho (Ac) - longitud cefalotorácica (Lc) & $\mathrm{Lc}=\mathrm{a}+\mathrm{b}^{*} \mathrm{Ac}$ & 241 & $108-166$ & $-0,805$ & 0,879 & 0,966 \\
\hline Longitud cefalotorácica (Lc) - peso total (Wt) & $\mathrm{Wt}=\mathrm{a}^{*} \mathrm{~L} c^{\mathrm{b}}$ & 594 & $88-141$ & 0,000961 & 2,817 & 0,943 \\
\hline Ancho cefalotorácico (Ac) - peso total (Wt) & $\mathrm{Wt}=\mathrm{a}^{*} \mathrm{Ac} \mathrm{c}^{\mathrm{b}}$ & 594 & $102-161$ & 0,000376 & 2,977 & 0,943 \\
\hline
\end{tabular}

(*) En hembras no se contó con una muestra representativa

abundancia se utilizó el valor de captura por trampa obtenida por trampas de diseño troncocónico con entrada de plástico, dado que éste fue el modelo determinado como más apropiado para la pesca de este recurso (Arana y Vega, 2000). Se considera que la CPUE obtenida con este aparejo es representativa de la abundancia en el correspondiente rango batimétrico de pesca e igualmente en todas las zonas alrededor de las islas. Dicha CPUE es de 7,1 kg/trampa u 8,2 ejemplares/trampa.

d) Radio de influencia del aparejo. Uno de los aspectos más preponderantes en la metodología de evaluación mediante el Area Teórica de Influencia de las Trampas, es la definición del radio de influencia del aparejo de pesca. De acuerdo a resultados de investigaciones en las que se ha utilizado técnicas fotográficas y otros medios de observación directa, se ha estimado el radio de pesca efectiva de 27 a $30 \mathrm{~m}$ al emplear trampas en la captura de cangrejos de aguas profundas de los géneros Geryon y Chaceon (Miller, 1975; McElman y Elner, 1982 y Defeo et al., 1990). Por tal motivo, se adoptó un radio de 30 $m$ en este estudio, al igual a como se han efectuado evaluaciones en experiencias similares en estos cangrejos. Así, también, se consideró que no existe interacción entre las trampas, ya que éstas quedaron distanciadas entre sí entre 100 y $200 \mathrm{~m}$.

e) Crecimiento. Se inspeccionaron las distribuciones de frecuencias de tallas obtenidas y se determinaron las posibles modas en dicha distri- bución. Para ello se utilizó el programa computacional FiSAT (FAO/ICLARM Stock Assessment Tools), utilizando como antecedentes los valores de incrementos por muda señalados por Melville-Smith (1988b, 1989). Con esta información, se obtuvo los siguientes parámetros de crecimiento (Figs. 6 y 7):

$\mathrm{L}_{\circ}=150 \mathrm{~mm}$ de largo cefalotorácico

$\mathrm{W}_{\circ}=1297 \mathrm{~g}$ de peso total húmedo

$\mathrm{k}=0,1 \mathrm{año}^{-1}$

$\mathrm{t}_{0} \quad=\quad 0,5$ años

f) Mortalidad natural. Este valor se determinó mediante un proceso iterativo, de forma que el valor de mortalidad, en ausencia de pesca, permitiera que una mínima parte de organismos alcanzara el $95 \%$ de la longitud asintótica. Así, se estimó como valor más probable $\mathrm{M}=0,15$. Cabe hacer notar que este mismo valor fue utilizado por Defeo et al. (1990) en C. notiabilis, y Melville-Smith (1988b) en C. maritae.

La biomasa disponible de $C$. chilensis se estimó en 995,3 ton en el rango de profundidad de 175 a $625 \mathrm{~m}$ (Tabla 2), con un promedio general de 100 toneladas en cada estrato de 50 metros. La densidad promedio, utilizando el peso medio global de los ejemplares, corresponde a 2,9 ind./1000 $\mathrm{m}^{2}$.

Al efectuar la evaluación utilizando la captura promedio por trampa en número de ejemplares, se determinó la abundancia en 993.997 ejemplares (Ta- 
Tabla 2. Estimación de biomasa (en peso) y densidad (ind./1000 $\mathrm{m}^{2}$ ) de cangrejo dorado de Juan Fernández, mediante el método de área de influencia de las trampas.

Table 2. Golden crab biomass estimate (by weight) and densities (ind./1000 $\mathrm{m}^{2}$ ), by the trap area of influence method.

\begin{tabular}{|c|c|c|c|c|c|c|c|c|c|}
\hline \multirow[b]{2}{*}{$\begin{array}{l}\text { Rango de } \\
\text { profundidad } \\
\text { (m) }\end{array}$} & \multirow[b]{2}{*}{$\begin{array}{l}\text { Profundi- } \\
\text { dad media } \\
\text { (m) }\end{array}$} & \multirow[b]{2}{*}{$\begin{array}{c}\text { Superficie } \\
\text { total } \\
\left(\mathbf{m n}^{2}\right)\end{array}$} & \multirow[b]{2}{*}{$\begin{array}{c}\text { CPUE } \\
\text { media } \\
\text { (kg/ } \\
\text { trampa) }\end{array}$} & \multirow[b]{2}{*}{$\begin{array}{c}\text { CPUE } \\
\text { media } \\
\text { (ind/ } \\
\text { trampa) }\end{array}$} & \multirow[b]{2}{*}{$\begin{array}{c}\text { Area de } \\
\text { pesca de las } \\
\text { trampas } \\
\left(\mathbf{m}^{2}\right)\end{array}$} & \multicolumn{2}{|c|}{ Estimación en peso } & \multicolumn{2}{|c|}{ Estimación en número } \\
\hline & & & & & & $\begin{array}{c}\text { Biomasa } \\
\text { disponible } \\
\text { (ton) }\end{array}$ & $\begin{array}{c}\text { Densidad } \\
\text { pr omedio } \\
\text { (ind./ } \\
\left.1000 \mathrm{~m}^{2}\right)\end{array}$ & $\begin{array}{c}\text { Númer o } \\
\text { de } \\
\text { indi vi- } \\
\text { duos }\end{array}$ & $\begin{array}{c}\text { Densidad } \\
\text { pr omedio } \\
\text { (ind./ } \\
\left.1000 \mathbf{m}^{2}\right)\end{array}$ \\
\hline $176-225$ & 200 & 15,0 & 6,07 & 6,00 & 2827,4 & 110,5 & 2,0 & 109.177 & 2,1 \\
\hline $226-275$ & 250 & 16,9 & 6,07 & 6,07 & 2827,4 & 124,4 & 2,1 & 124.375 & 2,1 \\
\hline $276-325$ & 300 & 14,2 & 7,41 & 7,41 & 2827,4 & 127,7 & 2,7 & 127.669 & 2,6 \\
\hline $326-375$ & 350 & 11,5 & 6,69 & 6,69 & 2827,4 & 93,5 & 3,0 & 93.458 & 2,4 \\
\hline $376-425$ & 400 & 13,2 & 6,40 & 6,40 & 2827,4 & 102,7 & 2,9 & 102.683 & 2,3 \\
\hline $426-475$ & 450 & 14,9 & 5,06 & 5,06 & 2827,4 & 91,7 & 2,3 & 91.674 & 1,8 \\
\hline $476-525$ & 500 & 12,6 & 8,45 & 8,45 & 2827,4 & 128,7 & 3,8 & 128.695 & 3,0 \\
\hline $526-575$ & 550 & 10,2 & 9,44 & 9,44 & 2827,4 & 116,6 & 3,5 & 116.553 & 3,3 \\
\hline $576-625$ & 600 & 9,0 & 9,12 & 9,12 & 2827,4 & 99,7 & 3,2 & 99.713 & 3,2 \\
\hline TOTAL & & 117,5 & 7,08 & 8,20 & & 995,3 & 2,9 & 993.997 & 2,4 \\
\hline
\end{tabular}

Radio base de influencia de la trampa $\quad=\quad 30 \mathrm{~m}$

Peso medio de los ejemplares (machos) $\quad=863 \mathrm{~g}$

Radio (m) 30

Factor q 1

Factor área 1

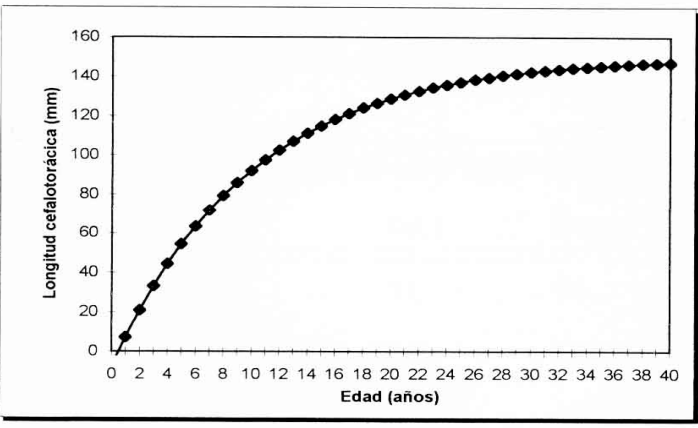

Figura 6. Curva teórica de crecimiento en longitud de machos de cangrejo dorado (Chaceon chilensis).

Figure 6. Theoretical length growth curve in male of golden crab (Chaceon chilensis).

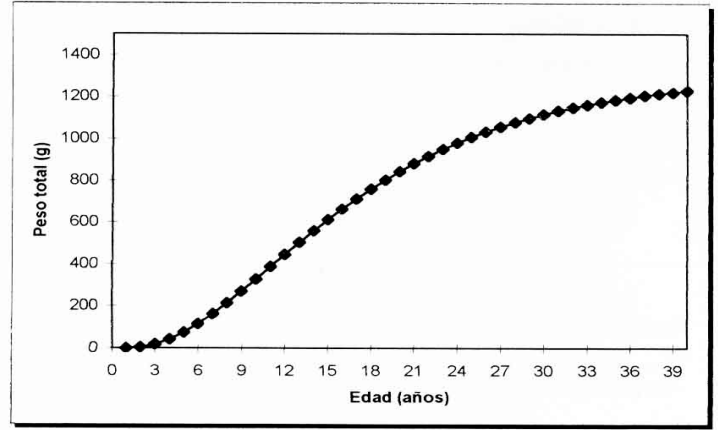

Figura 7. Curva teórica de crecimiento en peso de machos de cangrejo dorado (Chaceon chilensis).

Figure 7. Theoretical weight growth curve in male of golden crab (Chaceon chilensis). 
bla 2). En cada rango de 50 metros se estimó la presencia de aproximadamente 100.000 ejemplares. La abundancia así estimada permitió proyectar una densidad promedio global de 2,4 ind./1000 $\mathrm{m}^{2}$.

Los resultados fueron sensibilizaron respecto al radio de influencia de las trampas y al coeficiente de capturabilidad de las mismas (Tabla 3). En el caso del radio, se empleó con valores equivalentes al $50 \%$, $75 \%, 150 \%$ y $200 \%$, y el coeficiente de capturabilidad en $60 \%, 70 \%, 80 \%$ y $90 \%$. Como respuesta de este análisis, queda en evidencia la notable variación que se encuentra al considerar diversos valores en estas variables. Dentro del rango de sensibilización empleado, se obtuvieron biomasas comprendidas entre un mínimo de 500 ton y un máximo de 5.500 ton.

El rendimiento máximo sostenible (RMS) se determinó en 75 ton, utilizando la fórmula de Gulland (1971), considerando para ello el valor más probable de biomasa disponible y la mortalidad natural de $\mathrm{M}=0,15$ (Tabla 4). Dicho valor equivale a tres veces el desembarque que se efectúa anualmente 
Tabla 5. Densidades (ind./1000 $\mathrm{m}^{2}$ ) determinadas en cangrejos de los géneros Chaceon y Geryon.

Table 5. Crab genera Chaceon and Geryon densities (ind./1000 m²).

\begin{tabular}{|c|c|c|c|c|c|c|c|c|c|c|}
\hline \multirow[b]{2}{*}{ Autor, año } & \multirow[b]{2}{*}{ Especie } & \multirow[b]{2}{*}{ Método empleado } & \multirow[b]{2}{*}{ Area geográfica } & \multicolumn{7}{|c|}{ Rango de profundidad (m) } \\
\hline & & & & 200-299 & $300-399$ & $400-499$ & $500-599$ & $600-699$ & 700-799 & 800-899 \\
\hline Beyers y Wilke, 1980 & Geryon quinquedens & $\begin{array}{l}\text { Area barrida } \\
\text { red de arrastre }\end{array}$ & Suroeste de Africa & & $0,10-0,23$ & $0,10-0,64$ & $0,26-2,3$ & $0,15-0,75$ & $0,17-0,78$ & $0,45-0,75$ \\
\hline Melville-Smith, 1983 & Chaceon maritae & Fotografía & Suroeste de Africa & & & 2,25 & 4,42 & 4,82 & & \\
\hline Melville-Smith, 1988a & Chaceon maritae & $\begin{array}{l}\text { Area efectiva de } \\
\text { pesca (trampas) }\end{array}$ & Suroeste de Africa & & & 15,90 & 17,10 & 18,20 & 21,30 & 25,10 \\
\hline Melville-Smith, 1988a & Chaceon maritae & $\begin{array}{l}\text { Area barrida } \\
\text { red de arrastre }\end{array}$ & Suroeste de Africa & & 0,05 & 0,34 & 0,66 & 0,27 & 0,41 & 0,58 \\
\hline Wenner y Barans, 1990 & Chaceon fenneri & Fotografía & Sureste de USA & & & $0,16-0,64$ & $0,19-0,20$ & & & \\
\hline Presente trabajo & Chaceon chilensis & $\begin{array}{l}\text { Area efectiva } \\
\text { de pesca (trampas) }\end{array}$ & $\begin{array}{l}\text { Archipiélago de } \\
\text { Juan Fernández }\end{array}$ & $2,10-2,70$ & $2,90-3,00$ & $2,30-3,80$ & $3,20-3,50$ & & & \\
\hline
\end{tabular}


Tabla 6. Capturas de cangrejos de profundidad de los géneros Chaceon y Geryon, obtenidas con trampas en 24 horas de reposo.

Table 6. Crab genera Chaceon and Geryon catch by traps during 24 hour settings.

\begin{tabular}{|c|c|c|c|}
\hline Autor, año & Especie & Area geográfica & $\begin{array}{l}\text { Captura promedio } \\
\text { or trampa (kg/trampa) }\end{array}$ \\
\hline Cayré et al., 1979 & Geryon quinquedens & Costa de Marfil & 3 \\
\hline Cayré et al., 1979 & Geryon quinquedens & Congo & 6 \\
\hline Cayré et al., 1979 & Geryon quinquedens & Sur de Angola & 1,5 \\
\hline Elner y Robichaud, 1985 & Geryon quinquedens & Plataforma de Escocia & 16,7 \\
\hline Defeo et al., 1990 & Chaceon notiabilis & Uruguay & $4,9-10,3$ \\
\hline Holmsen, 1974 & Geryon quinquedens & Nueva Inglaterra, USA & 25 \\
\hline Intes, 1978 & Geryon quinquedens & Nueva Caledonia del Sur & 4,44 \\
\hline Kendall, 1990 & Chaceon affinis & Georgia, USA & 7 \\
\hline Poupin et al., 1991 & Chaceon sp. nov. & Islas Marquesas & 11,5 \\
\hline Wenner, 1990 & Chaceon fenneri & Costa oeste de USA & $2,0-10,0$ \\
\hline Wenner et al., 1987 & Chaceon fenneri & Costa oeste de USA & $1,7-18,0$ \\
\hline Lima y Lima Branco, 1991 & Geryon quinquedens & Sur de Brasil & 6,3 \\
\hline Arana y Venturini, 1991 & Chaceon chilensis & Cordillera de Nazca & 2,0 \\
\hline Presente investigación & Chaceon chilensis & Archipiélago de Juan Fernández & 7,1 \\
\hline
\end{tabular}

de la langosta de Juan Fernández. En el escenario más pesimista se obtiene un rendimiento máximo sostenible de 19 ton, mientras que el más optimista alcanza a 168 ton.

\section{DISCUSION}

La presencia de $C$. chilensis fue descrita inicialmente por Conan (1975), en faenas de pesca exploratoria realizadas en el cordón submarino del archipiélago de Juan Fernández. En torno a las islas Robinson Crusoe y Santa Clara se capturan ocasionalmente estos cangrejos cuando las trampas son caladas en forma excepcional a más de $180 \mathrm{~m}$ de profundidad. Los pescadores que también efectúan en forma esporádica faenas extractivas de langosta (Jasus frontalis) en las islas Desventuradas (islas San Félix y San Ambrosio), indican que aparecen ejemplares cuando calan trampas a profundidades mayores de $200 \mathrm{~m}$. En algunas oportunidades también se captura en aguas profundas del margen continental de Chile (Báez y Andrade, 1977). Así mismo, se encuentran cangrejos de este género en la cordillera de Nazca, donde se han extraído en faenas de pesca dirigidas a la langosta enana o dalmacita (Projasus bahamondei George, 1976) (Arana y Venturini,
1991).

Estos cangrejos de profundidad se explotan con cierta intensidad en diversos lugares del mundo. Así, Geryon quinquedens es pescado en la costa oeste de los Estados Unidos, con 1440 ton en 1993 y 465 ton en 1996; C. notiabilis en Uruguay, con 852 ton en 1994 y 1513 ton en 1996; por su parte Japón registra capturas de Geryon spp. con 6781 ton en 1993 y 4050 ton en 1996; C. maritae frente a la costa de Angola con 470 ton, y, en el cono sur de Africa tanto en el océano Atlántico como Indico se extraen 6.000 ton de C. maritae (FAO, 1996).

Respecto a los parámetros de crecimiento de $C$ chilensis, los únicos trabajos relativos al crecimiento de cangrejos de esta familia han sido realizados en $C$. maritae, del suroeste de Africa, mediante la técnica de marcaje y recaptura (Melville-Smith, 1988a, 1989). Los resultados demuestran que estos crustáceos crecen lentamente y que, en ejemplares adultos, la muda se produciría cada dos años o aún más espaciada.

La longitud asintótica determinada en este estudio $(\mathrm{Lc}=150 \mathrm{~mm})$ coincide con la longitud cefalotorácica máxima $(147 \mathrm{~mm})$ encontrada tanto por Arana y Vega (2000) en la pesca experimental realizada en el archipiélago de Juan Fernández, como por Arana y Venturini (1991), en la cordillera de 


\section{Nazca $(150 \mathrm{~mm})$.}

Al emplear el método de estimación de abundancia mediante el Area Teórica de Influencia de las Trampas, es posible identificar diversos sesgos que dependerán de la precisión con que se efectúe el cálculo del área de distribución del recurso; que el radio teórico de acción supuesto para las trampas se aproxime a la realidad; que la forma que adopta el área de influencia del aparejo sea circular, con escasa o nula superposición entre trampas colindantes; que la densidad del recurso sea homogénea en el área que se evalúa; y, que la captura de las trampas esté en proporción con la densidad del recurso en el área de pesca, siendo ésta representativa del estrato o subárea utilizado para efectuar la evaluación. De allí que los resultados obtenidos deben ser considerados como preliminares, aunque orientadores sobre la abundancia y biomasa de $C$ chilensis en el área de estudio.

De acuerdo a la probable área de influencia de las trampas, se determinaron densidades que fluctuarían entre 2,1 y 3,5 ejemplares por cada $1000 \mathrm{~m}^{2}$ de fondo marino (Tabla 5). Esto indicaría que la densidad de $C$. chilensis sería similar a la determinada en $C$. maritae en el sureste de Africa mediante análisis fotográfico (Melville-Smith, 1883) y mayor a la encontrada en $C$. fenneri por Wenner y Barans (1990) $\left(0,1-0,7\right.$ ind./1000 $\left.\mathrm{m}^{2}\right)$ en la costa sureste de EEUU, esta última realizada mediante observaciones submarinas. La alta densidad, así como la elevada proporción de ejemplares de gran talla encontrados en esta oportunidad, puede estar asociada al hecho de ser ésta una pesquería virgen.

Los rendimientos de pesca obtenidos en torno a las islas Robinson Crusoe y Santa Clara están igualmente en concordancia con valores máximos y mínimos encontrados en otros lugares (Tabla 6). Con excepción de las altas CPUE determinadas frente a la costa de Nueva Inglaterra y en la plataforma de Escocia, el rango de captura de cangrejos de los géneros Chaceon y Geryon está registrada entre 1,5 y $18,0 \mathrm{~kg} / \mathrm{trampa}$. Esto posiciona en un nivel intermedio los $7 \mathrm{~kg} /$ trampa determinados en estas experiencias como promedio general en la captura de $C$. chilensis en torno a estas islas al utilizar los diseños de trampas con los cuales se obtuvieron los rendimientos más altos. Estos corresponden a las trampas troncocónicas con túnel de plástico $(7,1 \mathrm{~kg} /$ trampa) y a la langostera con entrada en la parte superior $(5,1 \mathrm{~kg} /$ trampa $)$, diseño que es utilizado actualmente por los pescadores artesanales para la extracción de J. frontalis (Arana y Vega, 2000).

En relación a las capturas, destaca la baja cantidad de hembras obtenidas. No obstante, en investigaciones realizadas en otras partes del mundo se ha encontrado una característica similar (McElman y Elner, 1982; Wenner et al., 1987; Kendall, 1990). Así, Kendall (1990), de 3096 ejemplares capturados entre 240 y $490 \mathrm{~m}$ de profundidad, sólo el 2,5\% de los individuos correspondieron a hembras. En general, mediante el empleo de trampas se obtienen mayoritariamente machos de tallas comercializables. Según Barea y Defeo (1986), este fenómeno obedece a dos motivos: el tiempo de reposo de las trampas permitiría el escape de los ejemplares pequeños $y$, por otro lado, las hembras generalmente no penetran en las trampas debido a su poca actividad, hecho constatado por experiencias realizadas en laboratorio. En estas se verificó una búsqueda activa del alimento por parte de los machos, mientras que las hembras reaccionaban en forma mucho más lenta.

No obstante lo anterior, en faenas de pesca comercial realizadas en la cordillera submarina de Nazca, dirigidas a la extracción comercial de la langosta enana ( $P$. bahamondei), se capturaron conjuntamente con dicho recurso cangrejos de este género. En dicha oportunidad, el 48,6\% correspondió a hembras, de las cuales el $12 \%$ presentó la característica de hembras ovíferas (Arana y Venturini, 1991).

Las relaciones morfométricas establecidas en la presente investigación concuerdan en general con los resultados presentados por Trigg et al. (1997) respecto a $C$. fenneri y $C$. quinquedens del golfo de México. No obstante, en $C$. chilensis llama la atención la amplia dispersión en los pesos totales determinados en las tallas superiores, lo que queda en evidencia por el gran número de ejemplares pesados a esas longitudes. Tal diferencia puede estar asociada a la aparente lentitud de crecimiento establecida en estos cangrejos, dado lo cual se superpondrían las tallas correspondientes a diversas clases anuales.

En el caso que se inicie la explotación de $C$. chilensis en estas islas, los resultados obtenidos permiten prever la ausencia de problemas inmediatos asociados a la conservación de este recurso debido a que prácticamente no se capturan hembras, por lo que no sería necesario proteger este segmento de la población. Además, los cangrejos retenidos en las trampas son de gran talla, posiblemente la mayoría de ellos con longitudes superiores a la correspon- 
diente de primera madurez sexual. Al respecto, Trigg et al. (1997) mencionan que el Consejo Administrativo de las Pesquerías del Sudatlántico utilizan como talla mínima de captura para el cangrejo dorado de esa área, el equivalente a $105 \mathrm{~mm}$ de longitud cefalotorácica, lo que en el caso de $C$. chilensis correspondería a que más del $90 \%$ de los ejemplares capturados cumplirían con dicha talla mínima.

De acuerdo a los resultados obtenidos, se concluye que es factible explotar este recurso en niveles de cierta importancia por parte de los pescadores de esas islas, los que hasta ahora dirigen sus labores casi en forma exclusiva hacia la langosta $(J$. frontalis) y peces que constituyen la carnada de las trampas utilizadas para la extracción de este crustáceo. Si bien existe incertidumbre sobre los parámetros biológico-pesqueros y la evaluación de la abundancia y biomasa determinado en este estudio, debido a los métodos que son posibles de aplicar al efectuar las capturas con trampas, es factible iniciar la extracción con cantidades que se incrementen en forma escalonada en el tiempo. Esto permitiría monitorear el efecto de la pesca sobre este cangrejo y confirmar diversos aspectos relacionados con la biología de este recurso. Como primer horizonte de captura se podría definir un total de 20-25 ton/año, lo que representaría un desembarque equivalente al efectuado de langosta en la actualidad, lo que repercutiría positivamente en la economía insular y en beneficio directo de los pescadores que se dediquen a la extracción de este nuevo recurso.

\section{AGRADECIMIENTOS}

El autor agradece en forma especial al Técnico Muestreador Sr. Reinaldo Rehhof D. y a los Sres. Arturo Báez C., dueño de la chalupa de alta mar "Miriam" y Pedro S. Arredondo C., Patrón de Pesca, por su colaboración en la recolección de información y labores realizadas durante esta fase de la investigación. Así, también, reconoce agradecido las sugerencias de los tres evaluadores anónimos, destacando la prolija y acertada labor de revisión que realizó uno de ellos al documento preliminar.

\section{REFERENCIAS}

Andrade, H. 1985. Crustáceos decápodos marinos del archipiélago de Juan Fernández. En: P. Arana (ed.). Investigaciones Marinas en el Archipiélago de Juan Fernández. Esc. Ciencias del Mar, UCV, 109-116.

Arana, P. 2000. Pesca exploratoria con trampas alrededor de las islas Robinson Crusoe y Santa Clara, archipiélago de Juan Fernández, Chile. Invest. Mar., Valparaíso, 28: 39-52.

Arana, P. y R. Vega. 2000. Pesca experimental del cangrejo dorado (Chaceon chilensis) en el archipiélago de Juan Fernández, Chile. Invest. Mar., Valparaíso, 28: 69-81.

Arana, P. y V. Venturini. 1991. Investigaciones biológico-pesqueras de crustáceos en la cordillera submarina de Nazca (Océano Pacífico Suroriental). Inf. téc. Pesca Chile, 47: 1-86.

Arena, G., L. Barea y O. Defeo. 1988. Desarrollo de una metodología de evaluación mediante el uso de nasas. Publ. Com. Téc. Mix. Fr. Mar., 4: 55-66.

Báez, P. y H. Andrade. 1977. Geryon affinis Milne Edwards y Bouvier 1894 frente a las costas de Chile (Crustacea, Decapoda, Brachyura, Geryonidae). An. Mus. Hist. Nat., Valparaíso, 10: 215-219.

Báez, P. y R. Ruiz, 1985. Crustáceos de las islas oceánicas de Chile depositados en el Museo Nacional de Historia Natural de Santiago. En: P. Arana (ed.). Investigaciones Marinas en el Archipiélago de Juan Fernández. Esc. Ciencias del Mar, UCV, 93-108.

Barea, L. y O. Defeo. 1986. Aspectos de la pesquería del cangrejo rojo (Geryon quinquedens) en la Zona Común de Pesca Argentino-Uruguaya. Publ. Com. Téc. Mix. Fr. Mar., 1(1): 38-46.

Beyers, C.J. de B. y C.G. Wilke. 1980. Quantitative stock survey and some biological and morphometric characteristics of the deep-sea red crab Geryon quinquedens off South West Africa. Fish Bull. S. Afr., 13: 9-19.

Boschi, E.E., D.A. Bertuche, y J.G. Wyngaard. 1984. Estudio biológico pesquero de la centolla (Lithodes antacticus) del Canal Beagle, Tierra del Fuego, Argentina. Contrib. INIDEP. Primera parte. Instituto Nacional de Investigación y Desarrollo Pesquero, Mar del Plata, 1-72.

Cayré, P., P. Le Loueff y A. Intes. 1979. Geryon quinquedens, le crabe ruoge profond. Biologie, pêche, conditionnement, potentialités d'exploitation. Pêche Maritime, 1210: 1-8. 
Chirino-Gálvez, L.A. 1985. Stenotermia y biogeografía de geriónidos (Crustacea, Decapoda, Brachyura). Archivos de Biología y Medicina Experimentales, 18(2): R126 (resumen).

Chirino-Gálvez, L.A. y R.B. Manning. 1989. A new deep-sea crab of the genus Chaceon from Chile (Crustacea, Decapoda, Geryonidae). Proceedings of the Biological Society of Washington, 102(2): 401-404.

Comisión para la Conservación de los Recursos Vivos Marinos Antárticos (CCRVMA). 1992. Informe de la undécima reunión del Comité Científico. Comisión para la Conservación de los Recursos Vivos Marinos Antárticos, Horbat, Australia, 26-30 de octubre de 1992, 513 pp.

Conan, G.Y. 1975. Pesca exploratoria con trampas en cerros submarinos del archipiélago de Juan Fernández. CIMAR, UCV, no publicado.

Dawson, E.W. y W.R. Webber. 1991. The deep-sea crab Chaceon («Geryon»): a guide to information and a reference list of the family Geryonidae. National Museum of New Zealand Miscellaneous Series, 24: 1-83.

Defeo, O., V. Little y L. Barea. 1990. Estimaciones del stock del cangrejo rojo (Geryon quinquedens) en la Zona Común de Pesca Argentino-Uruguaya. Frente Marítimo, 6 (Secc. A): 53-66.

Dupré, E. 1975. Lista de crustáceos decápodos citados para el archipiélago de Juan Fernández. CIMAR, Documento Técnico, 8/75: 1-46.

Eggers, D.M., N.A. Rickard, D.G. Chapman y R.R. Whitney. 1982. A methodology for estimating area fished for baited hooks and traps along a ground line. Can. J. Fish. Aquat. Sci., 39: 448-453.

Elner, R.W. y D.A. Robichaud. 1985. The Scotian shelf fisheries for jonah crab Cancer borealis, and deep-sea red crab, Geryon quinquedens, 1984. CAFSAC Res., 85/6: 1-23.

Gulland, J. 1971. The fish resources of the ocean. West Byfleet, Surrey, Fishing News (Books), Ltd., London, $255 \mathrm{pp}$.

Holmsen, A.A. 1974. New England fishes with a future. Some under-exploited species. Maritimes, 18(1): 9-12.
Intes, A. 1978. Deep water pot fishing in New Caledonia and adjacent island: first results. The South Pacific Commisssion Fisheries Newsletter, 17: 10-12.

Kendall, D. 1990. An assessment of the Georgia golden crab fishery. In: W.J. Lindberg y E.L. Wenner (eds.), Geryonid crabs and associated continental slope fauna: a research workshop report. South Carolina Sea Grant Consortium, Florida Sea Grant College Program Technical Paper, 58: 18$19 \mathrm{pp}$.

Lima, J.H.M. de y R.L. Branco. 1991. Análise das operações de pesca do canranguejo de profundidade (Geryon quinquedens Smith 1879) por barcos japoneses arrendados na região sul do Brasil - 1984/85. Atlãntica, Rio Grande, 13(1): 179-187.

McElman, J.F. y R.W. Elner. 1982. Red crab (Geryon quinquedens) trap survey along the edge of the Scotian shelf, September 1980. Can. Tech. Rep. Fish. Aq. Sci., 1084: 1-12.

Melville-Smith, R. 1983. Some observations on the abundance of deep sea red crab Geryon maritae in South West African waters using photography. S. Afr. J. mar. Sci., 79(4): 1-144.

Melville-Smith, R. 1986. Red crab (Geryon maritae) density in 1985 by the technique of effective area fished per trap on the nortnern fishing grounds of South West Africa. S. Afr. J. mar. Sci., 4: 257-263.

Melville-Smith, R. 1988a. Comparative population size estimates for a portion of the red crab Geryon maritae stock off South West African coast. S. Afr. J. mar. Sci., 6: 23-31.

Melville-Smith, R. 1988b. The commercial fishery for and population dynamics of red crab Geryon maritae off South West Africa, 1976-1986. S. Afr. J. mar. Sci., 6: 79-95.

Melville-Smith, R. 1989. A growth model for the deepsea red crab (Geryon maritae) off South West Africa/Namibia (Decapoda, Brachyura). Crustaceana, 53(3): 279-292.

Miller, R.J. 1975. Density of the commercial spider crab, Chionoecetes opilio, and calibration of effective area fished per trap using bottom photography. J. Fish. Res. Board Can., 32(6): 761- 
768.

Organización de Naciones Unidas para la Agricultura y la Alimentación (FAO). 1996. Estadísticas de pesca. FAO, Estadísticas de pesca, 82: 700 pp.

Poupin, J., P. Buat y T. Ellis. 1991. Les crabes profonds des Iles Marquises (Chaceon sp. nov. Decapoda - Geryonidae). Service Mixte Control Biologique, Rapport Scientifique et Tech-nique, 40: 1-21.

Retamal, M.A. 1977. Sobre Geryon quinquedens Smith, 1870, en el Archipiélago de Juan Fernández. Bol. Soc. Biol. de Concepción, 51(1): 249-251.

Retamal, M.A. 1981. Catálogo ilustrado de los crustáceos decápodos de Chile. Gayana (zool.), 44: 1110.
Trigg, C., H. Perry y W. Brehm. 1997. Size and weight relationships for the golden crab, Chaceon fenneri, and the red crab, Chaceon quinquedens, from the eastern Gulf of Mexico. Gulf Research Reports, 9(4): 339-343.

Wenner, E.L. 1990. Distribution and abundance of golden crab, Chaceon fenneri, in the South Atlantic Bight. In: W.J. Lindberg y E.L. Wenner (eds.), Geryonid crabs and associated continental slope fauna: a research workshop report. South Carolina Sea Grant Consortium, Florida Sea Grant College Program Technical Paper, 58: 6-7.

Wenner, E.L. y C.A. Barans. 1990. In situ estimates of density of golden crab, Chaceon fenneri, from habitats on the continental slope, southeastern U.S. Bull. Mar. Sci., 46(3): 723-734.

Wenner, E.L., G.F. Ulrich and J.B. Wise. 1987. Exploration for golden crab, Geryon fenneri, in the South Atlantic bight: distribution, population structure, and gear assessment. US Fish. Bull., 85(34): 547-560. 